\title{
Association between indicators of systemic inflammation biomarkers during puberty with breast density and onset of menarche
}

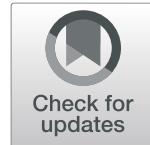

\author{
Karin B. Michels ${ }^{1,2^{*}}$ (D) Kristen Keller ${ }^{3}$, Ana Pereira ${ }^{4}$, Claire E. Kim ${ }^{1}$ José L. Santos ${ }^{5}$, John Shepherd ${ }^{6}$, \\ Camila Corvalan ${ }^{4}$ and Alexandra M. Binder ${ }^{1,6}$
}

\begin{abstract}
Background: Systemic inflammation may play a role in shaping breast composition, one of the strongest risk factors for breast cancer. Pubertal development presents a critical window of breast tissue susceptibility to exogenous and endogenous factors, including pro-inflammatory markers. However, little is known about the role of systemic inflammation on adolescent breast composition and pubertal development among girls.

Methods: We investigated associations between circulating levels of inflammatory markers (e.g., interleukin-6 (IL-6), tumor necrosis factor receptor 2 (TNFR2), and C-reactive protein (CRP)) at Tanner stages 2 and 4 and breast composition at Tanner stage 4 in a cohort of 397 adolescent girls in Santiago, Chile (Growth and Obesity Cohort Study, 2006-2018). Multivariable linear models were used to examine the association between breast composition and each inflammatory marker, stratifying by Tanner stage at inflammatory marker measurement. Accelerated failure time models were used to evaluate the association between inflammatory markers concentrations at each Tanner stage and time to menarche.
\end{abstract}

Results: In age-adjusted linear regression models, a doubling of TNFR2 at Tanner 2 was associated with a 26\% (95\% $\mathrm{Cl} 7-48 \%$ ) increase in total breast volume at Tanner 4 and a 22\% (95\% Cl 10-32\%) decrease of fibroglandular volume at Tanner 4. In multivariable models further adjusted for body fatness and other covariates, these associations were attenuated to the null. The time to menarche was 3\% (95\% Cl 1-5\%) shorter among those in the highest quartile of IL-6 at Tanner 2 relative to those in the lowest quartile in fully adjusted models. Compared to those in the lowest quartile of CRP at Tanner 4, those in the highest quartile experienced $2 \%(95 \% \mathrm{Cl} 0-3 \%)$ longer time to menarche in multivariable models.

Conclusions: Systemic inflammation during puberty was not associated with breast volume or breast density at the conclusion of breast development among pubertal girls after adjusting for body fatness; however, these circulating inflammation biomarkers, specifically CRP and IL-6, may affect the timing of menarche onset.

Keywords: Systemic inflammation, Breast Cancer, Breast density, Menarche, Adiposity, Puberty, Interleukin-6, Tumor necrosis factor receptor 2, C-reactive protein

\footnotetext{
*Correspondence: k.michels@ucla.edu

'Department of Epidemiology, Fielding School of Public Health, University of

California, 650 Charles Young Drive South, Room 71-264 CHS, Los Angeles,

CA 90095, USA

${ }^{2}$ Institute for Prevention and Cancer Epidemiology, Faculty of Medicine and Medical Center, University of Freiburg, Freiburg im Breisgau, Germany

Full list of author information is available at the end of the article
}

(c) The Author(s). 2020 Open Access This article is licensed under a Creative Commons Attribution 4.0 International License, which permits use, sharing, adaptation, distribution and reproduction in any medium or format, as long as you give appropriate credit to the original author(s) and the source, provide a link to the Creative Commons licence, and indicate if changes were made. The images or other third party material in this article are included in the article's Creative Commons licence, unless indicated otherwise in a credit line to the material. If material is not included in the article's Creative Commons licence and your intended use is not permitted by statutory regulation or exceeds the permitted use, you will need to obtain permission directly from the copyright holder. To view a copy of this licence, visit http://creativecommons.org/licenses/by/4.0/ The Creative Commons Public Domain Dedication waiver (http://creativecommons.org/publicdomain/zero/1.0/) applies to the data made available in this article, unless otherwise stated in a credit line to the data. 


\section{Background}

Chronic inflammation is increasingly suspected to play a critical role in breast cancer risk and progression [1]. The association between chronic inflammation and tumorigenesis may be driven by an impact of inflammation on estrogen synthesis, as well as general cell proliferation, insulin resistance, and insulin-like growth factor I [2-4]. Notably, these downstream impacts of chronic inflammation may shape breast composition, one of the strongest predictors of breast cancer risk [5].

Pro-inflammatory markers, such as interleukin-6 (IL6), tumor necrosis factor $\alpha$ (TNF $\alpha$ ), and C-reactive protein $(\mathrm{CRP})$, elevate in response to infection, tissue damage, and in active stressed states such as obesity [6]. These persistent triggering factors may disrupt the balance between the expressions of pro- and antiinflammatory markers and initiate sustained subclinical systemic inflammation. Inflammatory markers are secreted by both inflammatory cells, but also normal and malignant mammary cells [4]. Overexpression of these markers may induce continuous cellular proliferation, genomic instability, and cellular membrane damage. Further, they may suppress the antitumor immune response and increase estrogen levels in breast tissue, which all may directly promote the steps of neoplastic transformation through increased breast density [7].

Prior studies suggest that increased levels of certain pro-inflammatory markers may stimulate breast tumor growth and proliferation. IL-6 is a pro-inflammatory cytokine that has been associated with both breast cancer risk and progression [8]. Tumor necrosis factor (TNF) receptor 2 (TNFR2) has also been shown to have pro-inflammatory effects and to initiate immune modulation as well as tissue regeneration [9]. TNFR2 is one of the two soluble receptors of the cytokines TNF and lymphotoxin- $\alpha$, and TNFR2 directly promotes the proliferation of tumor cells while activating immunosuppressive cells [10]. CRP, an acute-phase inflammatory marker, is produced in the liver along with IL-6 and TNF $\alpha$. Epidemiologic studies have found that elevated levels of CRP were associated with cancer progression by providing a permissive environment for recurrent tumor growth (notably in gastrointestinal and kidney malignancies and a few in breast malignancies), inducing DNA damage, and promoting angiogenesis [11].

One of the mechanisms by which these proinflammatory markers may be related to cancer risk is through an influence on estrogen production. IL-6 has been identified as a stimulating factor for aromatase, the enzyme responsible for estrogen production in adipose tissue via conversion of androstenedione to estrogen [4]. Overexpression of these cytokines influences the growth and progression of malignant epithelial cells [12]. Similar to IL-6, increased circulating levels of TNFR2 may influence breast cancer risk through its effect on the estrogen pathway. TNF- $\alpha$ increases the production of aromatase, which may lead to greater breast density [13]. While CRP's role in the pathogenesis of cancer remains elusive, it has been suggested to contribute to the growth of breast density by stimulating local estrogen production [14]. The Study of Women's Health Across the Nation (SWAN) study, a longitudinal cohort study examining CRP and mammographic density, reported that high levels of CRP were associated with a slower decline in percent breast density with age [15].

The relation between pro-inflammatory markers and estrogen production may also have implications for pubertal development, another key predictor of breast cancer risk [16]. Pubertal breast development follows a coordinated surge in adrenal hormones preceding the reactivation of hypothalamic-pituitary-ovarian axis and production of estrogen from the ovaries [17]. Peak breast density is then achieved near the end of puberty [18]. There is growing evidence that this period of rapid mammary development creates a critical window of exposure susceptibility that can shape future breast cancer risk. Slower pubertal tempo (i.e., a longer window) has been associated with both increased breast density in young women and increased breast cancer risk, independent of age of menarche $[16,19]$. We have previously reported that exposure to specific endocrine-disrupting chemicals during childhood and puberty is associated with an increase in adolescent breast density [20]. Although pro-inflammatory markers may also influence estrogen signaling, no study has evaluated the relation between pro-inflammatory marker levels during this window and pubertal breast development.

We therefore examined the association between circulating levels of inflammatory markers at Tanner stages 2 and 4 and breast composition at Tanner stage 4 , as well as age at menarche, in a cohort of adolescent girls in Santiago, Chile. We hypothesize this study will provide greater insight into the influence of pro-inflammatory markers on breast density, which may have implications for future breast cancer risk.

\section{Methods \\ Study population}

The present study includes 397 girls participating in the Growth and Obesity Cohort Study (GOCS) in Santiago, Chile, for whom blood samples were collected at Tanner stages 2 and/or 4 and breast density was measured at Tanner stage 4. The GOCS was initiated in 2006 and included 1190 children (515 girls) aged between 2.6 and 4 years in the National Nursery Schools Council Program in Santiago and are representative of low to middleincome Chilean children. Participants were singletons born at term (37-42 weeks), with a birth weight between 
2500 and $4500 \mathrm{~g}$, and free from conditions that could affect growth such as food allergies and genetic and metabolic diseases. Anthropometric, growth, and maturation assessments were performed at the Institute of $\mathrm{Nu}$ trition and Food Technology (INTA) Health Clinic approximately every 6 to 12 months, and biological specimens were collected at defined time points over followup. More details on recruitment procedures and study design have been published previously [21]. The study protocol was approved by the Ethics Committee of INTA, University of Chile. Written informed consent was obtained from all parents or guardians of children prior to the start of data collection. Additional written informed consent was received from a parent or guardian when the data collection protocol was revised. The children gave assent when they turned 7 years of age.

\section{Tanner staging and assessment of breast tissue density}

Breast tissue density measurements were collected at Tanner stage 4. Starting in 2010, breast development was assessed during clinical visits by visual inspection using Tanner's rating scale (Tanner), and for Tanner 2, by palpation by a single female dietitian (trained by a pediatric endocrinologist with a kappa $=0.9$ ) [22]. Breast composition was derived from a two-compartment model of adipose and fibro-glandular tissues using software developed by Shepherd and colleagues [23]. Breast fibroglandular volume (FGV; $\mathrm{cm}^{3}$ ), total breast volume $\left(\mathrm{BV} ; \mathrm{cm}^{3}\right)$, and fibroglandular volume \% $(\mathrm{FGV} \%=\mathrm{FGV} /$ BV $\times 100$ ) were measured in the left and right breast once girls reached Tanner stage 4 by dual-energy X-ray absorptiometry (DXA) with a protocol designed to quantify breast composition [23]. Each breast was scanned using the Prodigy DXA system (GE Healthcare, Madison, WI, USA) software version 13.6., series 200674. The dosage of radiation exhibited by this assessment is extremely low and is lower than that received during a transcontinental flight, limiting any significant health risks associated with this X-ray method [24]. The protocol does not require breast compression and its validity and precision for measuring breast density in girls at different Tanner stages has been demonstrated previously [18]. A quality control phantom containing reference breast density materials was scanned throughout the study to assure a stable calibration. Values of the left and right breast were averaged for all analyses.

\section{Processing of blood samples}

Inflammatory markers were measured via fasting blood samples $(10 \mathrm{~mL})$, which were collected at Tanner stages 2 and 4 . Inflammatory markers were measured at Tanner 2 and 4 stages to evaluate consistency in the relationship between inflammatory markers across pubertal stages and adolescent breast composition. Samples were centrifuged, and serum and buffy coat separated. Serum samples were stored at $-80^{\circ} \mathrm{C}$ until further processing.

\section{Assessment of inflammatory markers Interleukin-6 (IL-6)}

IL-6 was measured by Quantikine ${ }^{\circ}$ Colorimetric Sandwich high-sensitive ELISA assay from R \& D Systems, Minneapolis, MN (www.rndsystems.com). The assay employs the quantitative sandwich enzyme immunoassay technique. A monoclonal antibody specific for IL-6 has been pre-coated onto a microtitre plate. After the addition of samples, standards, controls, and conjugates to the wells, IL-6 is sandwiched between the immobilized antibody and the enzyme-linked antibody specific to IL-6. Upon the addition of a substrate, a color is generated that is proportional to the amount of IL- 6 present in the sample. The assay has a sensitivity of $0.094 \mathrm{pg} /$ $\mathrm{mL}$, and the day-to-day variabilities of the assay at concentrations of $0.49,2.78$, and $5.65 \mathrm{pg} / \mathrm{mL}$ are 9.6, 7.2, and $6.5 \%$, respectively.

\section{TNF receptor 2 (TNFR2)}

TNFR2 was measured by an ELISA assay from $R$ \& D Systems. The assay employs the quantitative sandwich enzyme immunoassay technique. A monoclonal antibody specific for TNFR2 has been pre-coated onto a microtitre plate. After the addition of samples, standards, controls, and conjugates to the wells, TNFR2 is sandwiched between the immobilized antibody and the enzymelinked antibody specific to TNFR2. Upon the addition of a substrate, a color is generated that is proportional to the amount of TNFR2 present in the sample. The assay has a sensitivity of $0.6 \mathrm{pg} / \mathrm{mL}$. The day-to-day variabilities of the assay at concentrations of 89.9, 197, and 444 $\mathrm{pg} / \mathrm{mL}$ are $5.1,3.5$, and $3.6 \%$, respectively.

\section{High sensitivity C-reactive protein (hsCRP)}

Serum levels of CRP were measured with a highsensitive kit (ELISA, Kit, sensitivity: $0.12 \mathrm{mg} / \mathrm{L}$, interassay coefficient variation: 6.3\%; BIOMERICA, Inc.) [25].

\section{Covariates}

Covariates included in our analyses were assessed at Tanner stages 2 and 4 . Weight and height were measured every 6-12 months during follow-up using standardized techniques by trained personnel as previously described [26]; height for age $z$-scores was calculated based on the World Health Organization (WHO) 2007 growth references [27]. Body fat percentage was estimated at each visit using Tanita-BC-418 MA bioelectrical impedance measurements (Tanita-Corporation, Tokyo, Japan), at a measurement frequency of $50 \mathrm{kHz}$ (accuracy $0.1 \mathrm{~kg}$ ) [28]. Information on birthweight (grams) was obtained from birth records. Participant 
ethnicity was categorized based on whether their parent's surnames were of Mapuche origin (e.g., no indigenous surnames vs. one or more indigenous surnames) [29]. Maternal education was considered an indicator of socio-economic status and categorized by whether the participant's mother self-reported any post-secondary education. We adjusted our statistical models for birthweight, height, fat percentage, ethnicity, and maternal education because they have been previously been associated with inflammatory markers [30-32]. These variables have also been associated with pubertal timing and development [33-35].

\section{Statistical analysis}

Multivariable linear models were used to examine the association between log-transformed breast composition and each inflammatory marker, stratifying by Tanner stage at inflammatory marker measurement. Associations between Tanner 2 inflammatory markers and breast composition were prospective, whereas Tanner 4 inflammatory markers and breast composition were measured concurrently. Associations with logtransformed inflammatory marker concentration were first estimated adjusting for age at inflammatory marker measurement ("age-adjusted model"). We then considered models further adjusting for fat percentage at biomarker measurement ("age and body fatness-adjusted model") and fully adjusted models including age and fat percentage at biomarker measurement, as well as ethnicity (no indigenous surnames vs. one or more indigenous surnames), birth weight, age- and sex-specific height $Z$-score, and maternal education ("multivariable-adjusted model"). For each inflammatory marker, we reported the relative change in each breast composition measurement per doubling of inflammatory marker concentration and corresponding 95\% confidence interval (CI). To accommodate more complicated dose-response relations, we alternatively modeled the association between logtransformed breast composition and indicator variables for quartiles of inflammatory measurement, adjusting for the same covariates. Quartile cut points were determined on the original scale in the study population, stratifying by Tanner stage at inflammatory marker measurement (i.e., quartile cut points are Tanner stage specific). Based on these models, we reported the relative change in each breast composition measurement for each quartile relative to the lowest quartile and corresponding $95 \% \mathrm{CI}$.

Accelerated failure time models were used to evaluate the association between serum inflammatory marker concentration and time to menarche, stratifying by Tanner stage at biomarker measurement. Assuming a Weibull distribution, time to menarche was calculated as the time from birth to self-reported age at menarche. For the analysis of Tanner 4 inflammatory markers, individuals were excluded if menarche occurred before biomarker measurement ( $n=39$ left-censored); no participants were left-censored in the analysis of Tanner 2 inflammatory markers. Follow-up time for rightcensored individuals was age at last visit. Similar to our models for breast composition, we modeled the association with log-transformed biomarker measurement, adjusting for the same covariates. We report the relative time to menarche (time ratio) per doubling of inflammatory marker and $95 \% \mathrm{CI}$. We also modeled the association between quartiles of biomarker measurement and the time to menarche. We report the estimated relative time to menarche for each quartile of inflammatory marker measurement compared to the first quartile. Wald tests were used to evaluate the statistical significance of the associations with inflammatory marker concentration when modeled as a continuous measure. When modeled categorically, a likelihood ratio test was used to evaluate whether the inclusion of inflammatory marker level quartiles significantly (alphalevel $=0.05)$ improved model fit relative to the model without indicators for inflammatory biomarker quartiles. To evaluate the statistical trend across quartiles, the log-transformed median within each quartile was included as a continuous covariate in the models. To compare whether the characteristics of those lost to follow-up after Tanner 2, or those who only consented to a blood draw at one of the Tanner stages, impact the estimated associations, sensitivity analyses were conducted restricting to the subset of individuals for which inflammatory markers were measured at both time points. All analyses were conducted in $\mathrm{R}$ version 3.6.2.

\section{Results}

\section{Description of study population}

Our study population at Tanner stage 2 included 397 girls with a mean age of 9.44 years, and 356 girls at Tanner 4 with a mean age of 11.16 years (Table 1). Of the 397 girls with inflammatory measurements at Tanner stage 2, 119 were lost to follow-up or did not consent to provide breast composition measurements at Tanner stage 4. These 119 participants tended to be older at the Tanner stage 2 visit, have a higher fat body fat percentage, and have higher levels of each inflammatory marker (Additional file 1). Very few participants provided a blood sample at Tanner stage 4 that did not consent to provide a breast composition measurement $(n=11)$. These 11 participants had slightly lower levels of the inflammatory markers, but otherwise had similar characteristics to those that provided breast composition measurements (Additional file 2). Their mean age at menarche was 11.8 years and 11.75 years, respectively. Plasma levels of CRP were measured 
Table 1 Characteristics of pubertal girls at Tanner 2 and Tanner 4 in the Chilean Growth and Obesity Cohort Study

\begin{tabular}{|c|c|c|c|c|}
\hline \multirow[t]{2}{*}{ Characteristic } & \multicolumn{2}{|c|}{ Tanner $2(n=397)$} & \multicolumn{2}{|c|}{ Tanner $4(n=356)$} \\
\hline & $N$ missing & Distribution $^{A}$ & $N$ missing & Distribution $^{A}$ \\
\hline Age (years) & 0 & $9.44(1.31)$ & 0 & $11.16(0.84)$ \\
\hline Age at menarche & 52 & $11.80(0.89)$ & 24 & $11.75(0.84)$ \\
\hline Height (Z-score) & 0 & $0.09(0.96)$ & 1 & $0.39(1.00)$ \\
\hline BMI (Z-score) & 0 & $0.81(1.12)$ & 1 & $0.91(1.05)$ \\
\hline Fat percentage (\%) & 1 & $26.28(4.88)$ & 0 & $26.57(5.06)$ \\
\hline Maternal education & 0 & & 0 & \\
\hline No post-secondary education & & $304(76.57)$ & & $268(75.28)$ \\
\hline Post-secondary education & & $93(23.43)$ & & $88(24.72)$ \\
\hline Ethnicity & 0 & & 0 & \\
\hline No Mapuche background & & $327(82.37)$ & & $294(82.58)$ \\
\hline Mapuche background & & $70(17.63)$ & & $62(17.42)$ \\
\hline Birth weight (kg) & 12 & $3.38(0.39)$ & 10 & $3.34(0.39)$ \\
\hline Birth length $(\mathrm{cm})$ & 12 & $49.77(1.71)$ & 10 & $49.68(1.74)$ \\
\hline \multicolumn{5}{|l|}{ Inflammatory biomarkers ${ }^{B}$} \\
\hline C-reactive protein (CRP; mg/L) & 44 & $1.74(2.46)$ & 2 & $1.49(2.26)$ \\
\hline Interleukin-6 (IL-6; pg/mL) & 11 & $2.10(2.70)$ & 5 & $1.93(2.29)$ \\
\hline TNF receptor 2 (TNFR2; pg/mL) & 11 & $2270.96(579.53)$ & 5 & $2345.29(548.80)$ \\
\hline \multicolumn{5}{|l|}{ Breast composition at Tanner 4} \\
\hline Total breast volume $\left(\mathrm{BV} ; \mathrm{cm}^{3}\right)$ & 119 & $206.81(95.65)$ & 11 & $221.48(100.79)$ \\
\hline Fibroglandular volume $\left(\mathrm{FGV} ; \mathrm{cm}^{3}\right)$ & 119 & $81.50(32.11)$ & 11 & 82.59 (33.78) \\
\hline Percent fibroglandular volume (\%FGV; $\mathrm{cm}^{3}$ ) & 119 & $43.38(16.05)$ & 11 & $41.36(16.66)$ \\
\hline
\end{tabular}

${ }^{\mathrm{A}}$ Mean (SD) for continuous measures; count (\%) for categorical measures

${ }^{B}$ Range of inflammatory marker levels at Tanner 2: CRP 0.1-15.7 mg/L, IL-6 0.3-19.7 pg/mL, TNFR2 944.4-4902.5. Range of inflammatory markers at Tanner 4: CRP $0.1-18.9 \mathrm{mg} / \mathrm{L}$, IL-6 0.3-24.0 pg/mL, TNFR2 $1345.8-4548.1 \mathrm{pg} / \mathrm{mL}$

in 238 girls at Tanner 2 and 271 at Tanner 4, with 237 having measurements at both time points. For IL- 6 and TNFR2, plasma levels were measured in 266 girls at Tanner 2 and 269 at Tanner 4, with 263 having measurements at both time points. Plasma levels for IL-6 ( 2.10 vs. $1.93 \mathrm{pg} / \mathrm{mL})$ and CRP ( 1.74 vs. $1.49 \mathrm{mg} / \mathrm{L})$ were slightly higher in Tanner 2 than Tanner 4, while the opposite was found for TNFR2 (2270 vs. $2345 \mathrm{pg} / \mathrm{mL}$ ) (Table 1). Correlations between the three inflammatory markers measured were low and marginally stronger in Tanner 4 compared to Tanner 2. Spearman correlations of the individual inflammatory markers at Tanner stage 2 vs. Tanner stage 4 were 0.31 and 0.36 for IL6, 0.27 and 0.22 for TNFR2, and 0.11 and 0.23 CRP, respectively. Levels of all three inflammatory markers were positively correlated with body fat percentage. Spearman correlations of individual inflammatory markers and body fat percentage were 0.31 and 0.36 for IL6, 0.27 and 0.22 for TNFR2, and 0.11 and 0.23 for CRP, at Tanner 2 and Tanner 4 respectively (Fig. 1). Fat percentage assessed by the Tanita scale was positively correlated with BV and inversely correlated with FGV; no correlation was observed with FGV\%.

\section{Associations with breast composition}

In age-adjusted linear regression models, several statistically significant associations were found between inflammation marker levels measured at Tanner 2 and Tanner 4 and BV and FGV\% (Table 2). For example, a doubling of TNFR2 at Tanner 2 was associated with a $26 \%(95 \%$ CI 7-48\%) increase in breast volume at Tanner 4, and a $22 \%$ (95\% CI 10-32\%) decrease of FGV\% at Tanner 4. However, after further adjustment for fat percentage estimated by bioimpedance, all associations were attenuated to the null (Table 2). Additional adjustment for ethnicity, birthweight, and age- and sex-specific $z$-score for height did not further change our estimated associations between inflammatory markers and breast composition (Table 2). These results indicate that body fat percentage was the most important confounder in both analyses: it had a strong positive correlation with BV at Tanner 2 and Tanner 4 (Spearman rho: 0.64 and 0.72, respectively) and a strong negative correlation with FGV\%, at Tanner 2 and Tanner 4 (Spearman rho: -0.72 and -0.82 , respectively; Fig. 2). When the inflammatory markers were categorized into quartiles, several statistically significant trends were observed between the 


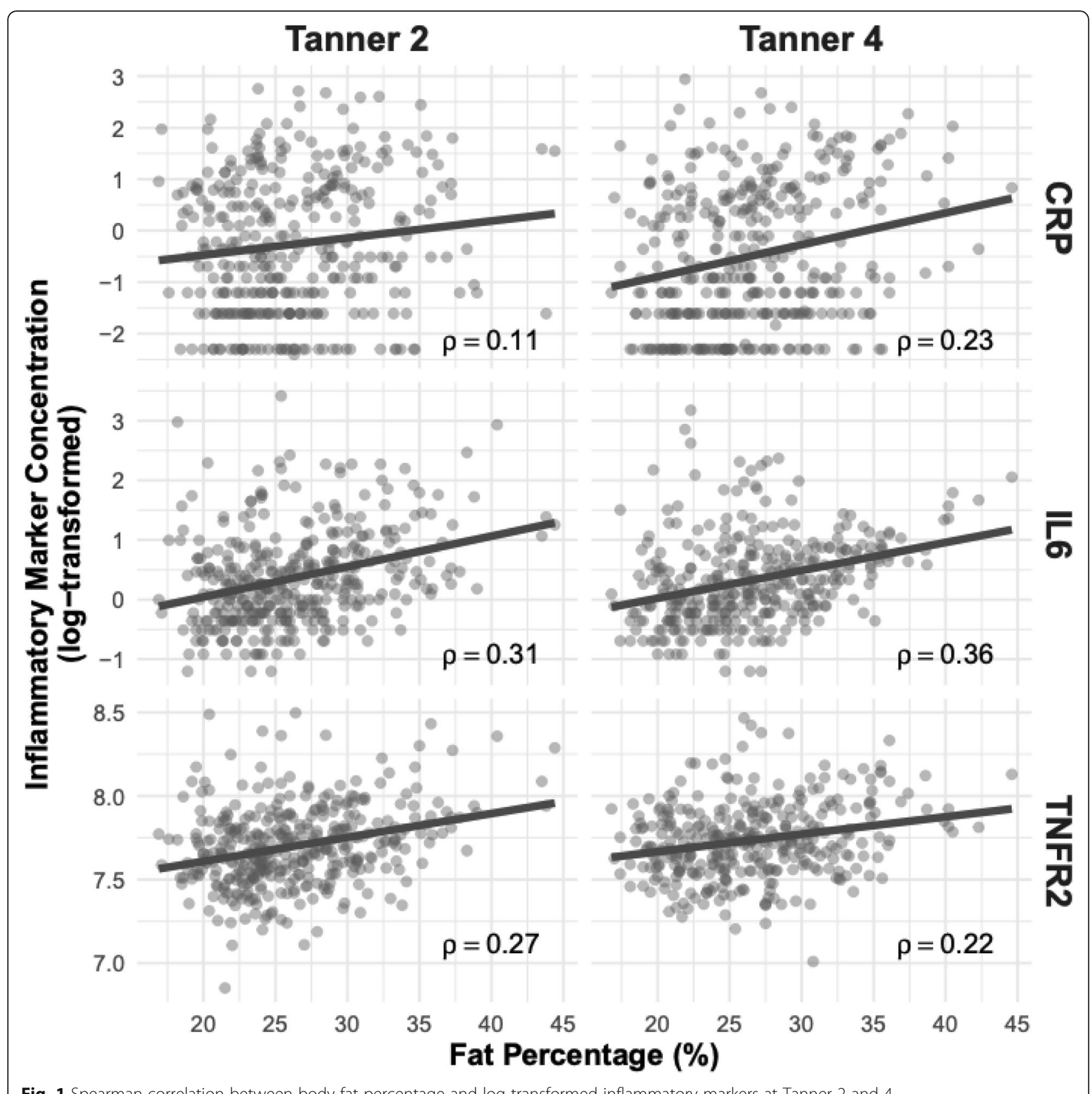

Fig. 1 Spearman correlation between body fat percentage and log-transformed inflammatory markers at Tanner 2 and 4

inflammation marker serum levels and BV and density measure, but none of these associations prevailed after adjustment for body fatness (Table 3). Sensitivity analyses were performed restricting to the subset of individuals for which inflammatory markers were measured at both time points to appraise whether the characteristics of those lost to follow-up after Tanner 2, or those who only consented to a blood draw at one of the Tanner stages, impact the estimated associations. The associations with breast composition and inference among this subset of the study did not differ from our primary results (Additional files 3, 4).

\section{Associations with age at menarche}

We also examined the association between inflammatory marker levels and the relative time to menarche from birth. A doubling of IL-6 levels at Tanner 2 was associated with a $1 \%(95 \%$ CI $0-1 \%)$ shorter time to menarche adjusting for age at biomarker measurement, which prevailed after adjustment for body fatness and other covariates (Table 4). This paralleled a 3\% (95\% CI 1-5\%) shorter time to menarche among girls in the highest quartile of IL-6 at Tanner 2 relative to girls in the lowest quartile (Table 5), corresponding to an approximately 3.8 months earlier median age of menarche, based on 
Table 2 Association of inflammatory markers measured at Tanner 2 and Tanner 4 with breast composition measured at Tanner 4

\begin{tabular}{|c|c|c|c|c|c|c|c|}
\hline \multirow{3}{*}{$\begin{array}{l}\text { Inflammatory } \\
\text { marker }\end{array}$} & \multirow{3}{*}{$\begin{array}{l}\text { Breast } \\
\text { Tanner } \\
\text { stage }\end{array}$} & \multicolumn{6}{|c|}{ Relative change in breast composition per doubling of inflammatory marker } \\
\hline & & \multicolumn{2}{|c|}{ Age-adjusted model $^{\mathrm{A}}$} & \multicolumn{2}{|c|}{ Age and body fatness-adjusted model ${ }^{B}$} & \multicolumn{2}{|c|}{ Multivariable-adjusted model $\mathrm{l}^{\mathrm{C}}$} \\
\hline & & $N$ & Estimate $(95 \% \mathrm{Cl})$ & $N$ & Estimate $(95 \% \mathrm{Cl})$ & $N$ & Estimate $(95 \% \mathrm{Cl})$ \\
\hline \multicolumn{8}{|c|}{ Total breast volume } \\
\hline \multirow[t]{2}{*}{ CRP } & Tanner 2 & 246 & $1.03(1.00-1.06)$ & 245 & $1.01(0.99-1.04)$ & 236 & $1.01(0.99-1.04)$ \\
\hline & Tanner 4 & 343 & $1.02(1.00-1.05)$ & 343 & $0.98(0.97-1.00)$ & 332 & $0.99(0.97-1.01)$ \\
\hline \multirow[t]{2}{*}{ IL-6 } & Tanner 2 & 272 & $1.05(1.00-1.10)^{*}$ & 271 & $0.98(0.94-1.02)$ & 262 & $0.98(0.94-1.02)$ \\
\hline & Tanner 4 & 341 & $1.10(1.06-1.15)^{* * *}$ & 341 & $1.01(0.97-1.04)$ & 330 & $1.01(0.97-1.04)$ \\
\hline \multirow[t]{2}{*}{ TNFR2 } & Tanner 2 & 272 & $1.26(1.07-1.48)^{* *}$ & 271 & $1.03(0.90-1.18)$ & 262 & $1.07(0.93-1.22)$ \\
\hline & Tanner 4 & 341 & $1.35(1.15-1.57)^{* * *}$ & 341 & $1.07(0.95-1.20)$ & 330 & $1.06(0.94-1.19)$ \\
\hline \multicolumn{8}{|c|}{ Fibroglandular volume } \\
\hline \multirow[t]{2}{*}{ CRP } & Tanner 2 & 246 & $1.00(0.97-1.03)$ & 245 & $1.00(0.97-1.03)$ & 236 & $1.00(0.98-1.03)$ \\
\hline & Tanner 4 & 343 & $0.98(0.96-1.00)^{*}$ & 343 & $0.98(0.96-1.00)^{*}$ & 332 & $0.98(0.96-1.01)$ \\
\hline \multirow[t]{2}{*}{ IL-6 } & Tanner 2 & 272 & $0.99(0.95-1.03)$ & 271 & $0.99(0.95-1.03)$ & 262 & $0.99(0.95-1.04)$ \\
\hline & Tanner 4 & 341 & $1.00(0.96-1.04)$ & 341 & $1.00(0.96-1.04)$ & 330 & $1.01(0.96-1.05)$ \\
\hline \multirow[t]{2}{*}{ TNFR2 } & Tanner 2 & 272 & $0.98(0.85-1.13)$ & 271 & $0.98(0.85-1.14)$ & 262 & $1.01(0.87-1.17)$ \\
\hline & Tanner 4 & 341 & $1.06(0.92-1.21)$ & 341 & $1.06(0.92-1.23)$ & 330 & $1.04(0.91-1.20)$ \\
\hline \multicolumn{8}{|c|}{ Percent fibroglandular volume } \\
\hline \multirow[t]{2}{*}{ CRP } & Tanner 2 & 246 & $0.97(0.95-0.99)^{*}$ & 245 & $0.99(0.97-1.01)$ & 236 & $0.99(0.97-1.01)$ \\
\hline & Tanner 4 & 343 & $0.96(0.94-0.98)^{* * *}$ & 343 & $0.99(0.98-1.01)$ & 332 & $1.00(0.98-1.01)$ \\
\hline \multirow[t]{2}{*}{$\mathrm{IL}-6$} & Tanner 2 & 272 & $0.94(0.90-0.98)^{* *}$ & 271 & $1.01(0.98-1.04)$ & 262 & $1.01(0.98-1.05)$ \\
\hline & Tanner 4 & 341 & $0.90(0.87-0.94)^{* * *}$ & 341 & $1.00(0.97-1.02)$ & 330 & $1.00(0.97-1.02)$ \\
\hline \multirow[t]{2}{*}{ TNFR2 } & Tanner 2 & 272 & $0.78(0.68-0.90)^{* * *}$ & 271 & $0.95(0.86-1.05)$ & 262 & $0.95(0.85-1.05)$ \\
\hline & Tanner 4 & 341 & $0.78(0.68-0.90)^{* * *}$ & 341 & $1.00(0.91-1.09)$ & 330 & $0.98(0.90-1.08)$ \\
\hline
\end{tabular}

A Linear regression model adjusting for age at inflammatory biomarker measurement

${ }^{B}$ Model adjusting for age at inflammatory biomarker measurement and fat percentage at biomarker measurement

${ }^{C}$ Model adjusting for age at inflammatory biomarker measurement, fat percentage at biomarker measurement, ethnicity, birth weight, height age- and sex-specific $Z$-score, and maternal education

${ }^{*} p<0.05$

$* * 0<0.01$

${ }^{* * *} p<0.001$

our multivariable model. Given our models estimated the change in the relative time to menarche, this change on the absolute scale only holds for the difference in the median age of menarche between the highest and lowest quartiles of Il- 6 at Tanner 2 and will differ for any other time point. We present this difference in months as an example of the implications at one specific time point. While Tanner 2 TNFR2 levels were also associated with time to menarche (Table 4), these associations did not persist when TNFR2 was modeled categorically (Table 5). A doubling of CRP at Tanner 4 was associated with a $1 \%$ (95\% CI 0-1\%) longer relative time to menarche, which also persisted after adjustment for covariates. These associations also manifested as significant trends when quartiles of inflammatory markers were modeled (Table 5). Notably, girls in the highest quartile of CRP at Tanner 4 had a $2 \%(95 \% \mathrm{CI} 0-3 \%)$ longer time to menarche relative to the first quartile, corresponding to an approximately
2.6 month later median age of menarche in fully adjusted models. Associations restricting to those for which inflammatory markers were measured at both time points were consistent with the associations observed in the full study population (Additional files 5, 6).

\section{Discussion}

To our knowledge, this is the first study of the association between circulating inflammatory markers and breast density among pubertal girls. Our results indicate that the association between inflammatory markers and adolescent breast density was driven by estimated body fat percentage. Regardless, our results are important to note as previous studies have found total BV to be associated with breast cancer risk among women with normal and lean body mass [36]. In contrast, the association between select inflammation markers and the onset of menarche persisted in our fully adjusted models. 


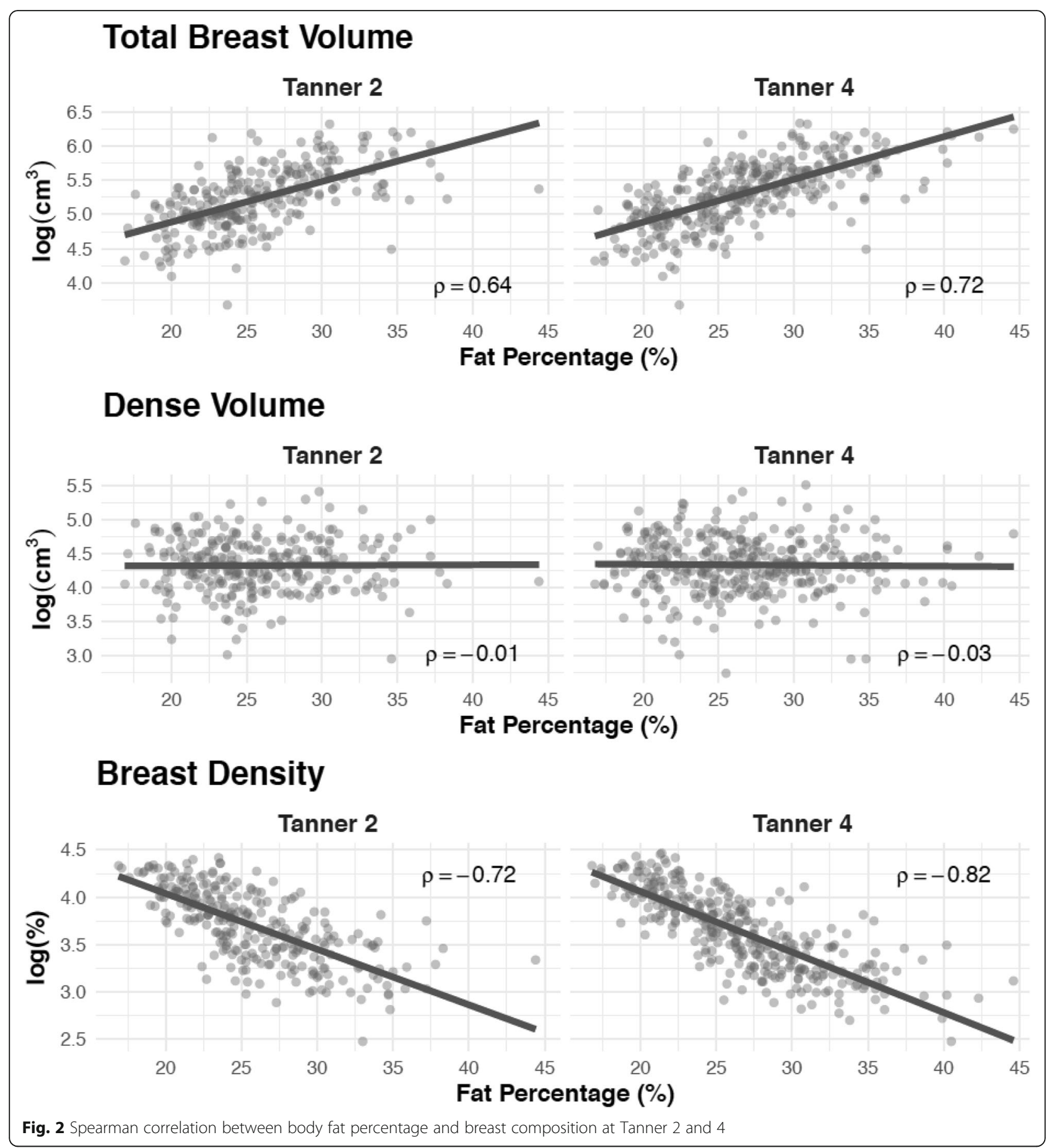

Our results reinforce the relation between systematic inflammation and adiposity [37]. Growing epidemiologic evidence suggests inflammation may mediate the association between adiposity and disease development [37-39]. Adipose tissue secretes a range of hormones, cytokines, chemokines, and growth factors, which can alter insulin sensitivity, glucose metabolism, estrogen production, and inflammation [40, 41]. Several prior studies among adolescents have shown an association between body fatness and markers of chronic inflammation (e.g., IL-6, TNF $\alpha$, and CRP) [42-45]. A recent study of adult female Chinese immigrants similarly found a positive association between CRP and TNFR2 and percent dense breast in unadjusted models [46]. For TNFR2, this association was similarly driven by non-dense breast area and was attenuated in adjusted models [46]. In contrast, CRP was 
Table 3 Association of quartiles of inflammatory markers measured at Tanner 2 and Tanner 4 with breast composition measured at Tanner 4

\begin{tabular}{|c|c|c|c|c|c|c|c|}
\hline \multirow{2}{*}{$\begin{array}{l}\text { Inflammatory } \\
\text { marker }\end{array}$} & \multirow{2}{*}{$\begin{array}{l}\text { Breast } \\
\text { Tanner } \\
\text { stage }\end{array}$} & \multirow[b]{2}{*}{$N$} & \multirow[b]{2}{*}{ LRT $p$ value ${ }^{\mathrm{D}}$} & \multicolumn{3}{|c|}{ Relative change in breast composition compared to Q1 $(95 \% \mathrm{Cl})^{\mathrm{F}}$} & \multirow[b]{2}{*}{$p$ for Trend ${ }^{E}$} \\
\hline & & & & Q2 & Q3 & Q4 & \\
\hline
\end{tabular}

\section{Age-adjusted model ${ }^{A}$}

Total breast volume

$\begin{array}{llll}\text { CRP } & \text { Tanner 2 } & \text { 246 } & 0.054 \\ & \text { Tanner 4 } & 343 & 0.199 \\ \text { IL-6 } & \text { Tanner 2 } & 272 & 0.036 \\ & \text { Tanner 4 } & 341 & <0.0001 \\ \text { TNFR2 } & \text { Tanner 2 } & 272 & 0.001 \\ & \text { Tanner 4 } & 341 & 0.002\end{array}$

$\begin{array}{ll}1.20(1.03-1.41)^{*} & 1.14(0.98-1.32) \\ 1.15(0.99-1.33) & 1.10(0.97-1.26) \\ 1.13(0.98-1.31) & 1.19(1.03-1.37)^{*} \\ 1.23(1.08-1.40)^{* *} & 1.27(1.11-1.45)^{* * *} \\ 1.02(0.88-1.18) & 1.23(1.06-1.42)^{* *} \\ 1.18(1.03-1.35)^{*} & 1.24(1.08-1.42)^{* *}\end{array}$

$\begin{array}{ll}1.19(1.02-1.38)^{*} & 0.037 \\ 1.11(0.98-1.26) & 0.131 \\ 1.22(1.05-1.42)^{*} & 0.010 \\ 1.41(1.24-1.61)^{* * *} & <0.0001 \\ 1.26(1.09-1.47)^{* *} & <0.001 \\ 1.29(1.12-1.48)^{* * *} & <0.001\end{array}$

Fibroglandular volume

$\begin{array}{llll}\text { CRP } & \text { Tanner 2 } & 246 & 0.759 \\ & \text { Tanner 4 } & 343 & 0.161 \\ \text { IL-6 } & \text { Tanner 2 } & 272 & 0.687 \\ & \text { Tanner 4 } & 341 & 0.156 \\ \text { TNFR2 } & \text { Tanner 2 } & 272 & 0.466 \\ & \text { Tanner 4 } & 341 & 0.585\end{array}$

$1.07(0.94-1.24)$
$0.99(0.87-1.13)$
$0.98(0.86-1.12)$
$1.03(0.91-1.16)$
$0.98(0.86-1.11)$
$1.06(0.94-1.20)$

$1.04(0.91-1.19)$

$1.01(0.89-1.16)$

0.840

0.99 (0.88-1.11)

$0.89(0.80-0.99)^{*}$

0.066

$0.96(0.85-1.09)$

$1.05(0.92-1.20)$

0.536

$0.89(0.79-1.01)$

$0.99(0.88-1.11)$

0.432

$1.08(0.95-1.23)$

$0.98(0.86-1.12)$

0.883

$1.09(0.96-1.23)$

$1.06(0.93-1.20)$

0.380

Percent fibroglandular volume

$\begin{array}{llll}\text { CRP } & \text { Tanner 2 } & \text { 246 } & 0.082 \\ & \text { Tanner 4 } & 343 & 0.001 \\ \text { IL-6 } & \text { Tanner 2 } & 272 & 0.005 \\ & \text { Tanner 4 } & 341 & <0.0001 \\ \text { TNFR2 } & \text { Tanner 2 } & 272 & <0.001 \\ & \text { Tanner 4 } & 341 & 0.017\end{array}$

$0.89(0.78-1.02)$
$0.87(0.76-0.99)^{*}$
$0.86(0.76-0.98)^{*}$
$0.84(0.75-0.94)^{* *}$
$0.96(0.85-1.09)$
$0.91(0.80-1.02)$

$0.92(0.81-1.04)$

$0.86(0.76-0.97)^{*}$

0.023

$0.90(0.80-1.01)$

$0.81(0.72-0.92)^{* * *}$

$0.80(0.72-0.90)^{* * *}$

$<0.001$

$0.86(0.76-0.98)^{*}$

0.018

$0.71(0.63-0.79)^{* * *}$

$0.70(0.63-0.78)^{* * *}$

$<0.0001$

$0.88(0.77-0.99)^{*}$

$0.78(0.69-0.89)^{* * *}$

$<0.0001$

$0.88(0.78-0.99)^{*}$

$0.82(0.72-0.93)^{* *}$

0.002

\section{Age and body fatness-adjusted model ${ }^{B}$}

Total breast volume

$\begin{array}{llll}\text { CRP } & \text { Tanner 2 } & 245 & 0.242 \\ & \text { Tanner 4 } & 343 & 0.043 \\ \text { IL-6 } & \text { Tanner 2 } & 271 & 0.986 \\ & \text { Tanner 4 } & 341 & 0.191 \\ \text { TNFR2 } & \text { Tanner 2 } & 271 & 0.344 \\ & \text { Tanner 4 } & 341 & 0.030\end{array}$

$1.13(1.00-1.29)$
$1.02(0.92-1.14)$
$1.01(0.90-1.14)$
$1.09(0.99-1.20)$
$1.00(0.89-1.12)$
$1.14(1.03-1.25)^{*}$

$1.09(0.96-1.23)$

$1.07(0.95-1.22)$

0.272

$1.02(0.93-1.12)$

$0.90(0.82-0.99)^{*}$

0.077

$1.02(0.90-1.15)$

$1.00(0.88-1.14)$

0.991

$0.98(0.88-1.09)$

$1.04(0.93-1.15)$

0.906

$1.10(0.97-1.24)$

$1.06(0.93-1.20)$

0.211

$1.14(1.03-1.26)^{*}$

$1.06(0.96-1.18)$

0.297

Fibroglandular volume

$\begin{array}{llll}\text { CRP } & \text { Tanner 2 } & \text { 245 } & 0.775 \\ & \text { Tanner 4 } & 343 & 0.146 \\ \text { IL-6 } & \text { Tanner 2 } & 271 & 0.694 \\ & \text { Tanner 4 } & 341 & 0.161 \\ \text { TNFR2 } & \text { Tanner 2 } & 271 & 0.478 \\ & \text { Tanner 4 } & 341 & 0.563\end{array}$

$$
\begin{aligned}
& 1.07(0.93-1.24) \\
& 0.99(0.87-1.12) \\
& 0.98(0.86-1.12) \\
& 1.02(0.91-1.16) \\
& 0.98(0.86-1.11) \\
& 1.07(0.94-1.20)
\end{aligned}
$$

$1.04(0.91-1.19)$

$1.01(0.88-1.16)$

0.884

$0.99(0.88-1.11)$

$0.88(0.79-0.99)^{*}$

0.060

$0.96(0.84-1.10)$

$1.05(0.91-1.21)$

0.540

0.89 (0.78-1.01)

$0.98(0.87-1.12)$

0.465

$1.08(0.94-1.23)$

$0.98(0.85-1.13)$

0.915

$1.09(0.96-1.24)$

$1.06(0.94-1.21)$

0.336

Percent fibroglandular volume

$\begin{array}{llll}\text { CRP } & \text { Tanner 2 } & 245 & 0.535 \\ & \text { Tanner 4 } & 343 & 0.750 \\ \text { IL-6 } & \text { Tanner 2 } & 271 & 0.169 \\ & \text { Tanner 4 } & 341 & 0.126\end{array}$

$0.94(0.86-1.04)$
$0.97(0.89-1.05)$
$0.97(0.88-1.06)$
$0.94(0.87-1.01)$

$0.95(0.87-1.05)$

$0.94(0.86-1.04)$

0.227

$0.96(0.90-1.04)$

$0.98(0.91-1.05)$

0.448

$0.94(0.86-1.04)$

$1.05(0.95-1.16)$

0.347

$0.91(0.84-0.98)^{*}$

$0.95(0.88-1.02)$

0.169 
Table 3 Association of quartiles of inflammatory markers measured at Tanner 2 and Tanner 4 with breast composition measured at Tanner 4 (Continued)

\begin{tabular}{|c|c|c|c|c|c|c|c|}
\hline \multirow{2}{*}{$\begin{array}{l}\text { Inflammatory } \\
\text { marker }\end{array}$} & \multirow{2}{*}{$\begin{array}{l}\text { Breast } \\
\text { Tanner } \\
\text { stage }\end{array}$} & \multirow[b]{2}{*}{$N$} & \multirow[b]{2}{*}{ LRT $p$ value ${ }^{\mathrm{D}}$} & \multicolumn{3}{|c|}{ Relative change in breast composition compared to Q1 $(95 \% \mathrm{Cl})^{\mathrm{F}}$} & \multirow[b]{2}{*}{$p$ for Trend ${ }^{E}$} \\
\hline & & & & Q2 & Q3 & Q4 & \\
\hline \multirow[t]{2}{*}{ TNFR2 } & Tanner 2 & 271 & 0.487 & $0.99(0.90-1.08)$ & $0.98(0.89-1.08)$ & $0.93(0.84-1.02)$ & 0.151 \\
\hline & Tanner 4 & 341 & 0.260 & $0.94(0.87-1.01)$ & $0.96(0.89-1.03)$ & $1.00(0.92-1.08)$ & 0.874 \\
\hline
\end{tabular}

Multivariable-adjusted model $^{\mathrm{c}}$

Total breast volume

\begin{tabular}{|c|c|c|c|c|c|c|c|}
\hline \multirow[t]{2}{*}{ CRP } & Tanner 2 & 236 & 0.314 & $1.12(0.98-1.28)$ & $1.08(0.96-1.23)$ & $1.08(0.95-1.22)$ & 0.234 \\
\hline & Tanner 4 & 332 & 0.247 & $1.03(0.93-1.14)$ & $1.02(0.93-1.12)$ & $0.94(0.85-1.03)$ & 0.286 \\
\hline \multirow[t]{2}{*}{ IL-6 } & Tanner 2 & 262 & 0.951 & $1.01(0.90-1.14)$ & $1.03(0.91-1.16)$ & $1.00(0.88-1.13)$ & 0.983 \\
\hline & Tanner 4 & 330 & 0.096 & $1.11(1.01-1.23)^{*}$ & $0.99(0.89-1.10)$ & $1.05(0.95-1.17)$ & 0.713 \\
\hline \multirow[t]{2}{*}{ TNFR2 } & Tanner 2 & 262 & 0.132 & $0.99(0.88-1.12)$ & $1.13(1.00-1.27)$ & $1.08(0.95-1.22)$ & 0.097 \\
\hline & Tanner 4 & 330 & 0.036 & $1.12(1.01-1.23)^{*}$ & $1.14(1.03-1.27)^{*}$ & $1.04(0.94-1.16)$ & 0.468 \\
\hline \multicolumn{8}{|c|}{ Fibroglandular volume } \\
\hline \multirow[t]{2}{*}{ CRP } & Tanner 2 & 236 & 0.787 & $1.07(0.93-1.23)$ & $1.05(0.92-1.20)$ & $1.03(0.90-1.18)$ & 0.695 \\
\hline & Tanner 4 & 332 & 0.556 & $0.99(0.88-1.13)$ & $0.99(0.88-1.11)$ & $0.92(0.82-1.04)$ & 0.236 \\
\hline \multirow[t]{2}{*}{ IL-6 } & Tanner 2 & 262 & 0.671 & $0.99(0.86-1.13)$ & $0.97(0.85-1.11)$ & $1.06(0.92-1.22)$ & 0.429 \\
\hline & Tanner 4 & 330 & 0.139 & $1.05(0.93-1.18)$ & $0.90(0.79-1.03)$ & $1.01(0.89-1.14)$ & 0.650 \\
\hline \multirow[t]{2}{*}{ TNFR2 } & Tanner 2 & 262 & 0.347 & $0.99(0.87-1.13)$ & $1.11(0.97-1.27)$ & $1.00(0.87-1.15)$ & 0.617 \\
\hline & Tanner 4 & 330 & 0.599 & $1.04(0.92-1.18)$ & $1.09(0.96-1.24)$ & $1.03(0.91-1.17)$ & 0.600 \\
\hline \multicolumn{8}{|c|}{ Percent fibroglandular volume } \\
\hline \multirow[t]{2}{*}{ CRP } & Tanner 2 & 236 & 0.721 & $0.96(0.87-1.06)$ & $0.97(0.88-1.07)$ & $0.95(0.87-1.05)$ & 0.340 \\
\hline & Tanner 4 & 332 & 0.769 & $0.97(0.89-1.05)$ & $0.96(0.90-1.04)$ & $0.98(0.91-1.06)$ & 0.579 \\
\hline \multirow[t]{2}{*}{ IL-6 } & Tanner 2 & 262 & 0.105 & $0.97(0.88-1.07)$ & $0.94(0.86-1.04)$ & $1.07(0.96-1.18)$ & 0.236 \\
\hline & Tanner 4 & 330 & 0.134 & $0.94(0.87-1.02)$ & $0.91(0.84-0.99)^{*}$ & $0.95(0.88-1.03)$ & 0.226 \\
\hline \multirow[t]{2}{*}{ TNFR2 } & Tanner 2 & 262 & 0.486 & $1.00(0.91-1.10)$ & $0.98(0.89-1.08)$ & $0.93(0.84-1.03)$ & 0.166 \\
\hline & Tanner 4 & 330 & 0.296 & $0.93(0.86-1.01)$ & $0.95(0.88-1.03)$ & $0.98(0.91-1.07)$ & 0.893 \\
\hline
\end{tabular}

${ }^{\mathrm{A}}$ Linear regression model adjusting for age at inflammatory biomarker measurement

${ }^{\mathrm{B}}$ Model adjusting for age at inflammatory biomarker measurement and fat percentage at biomarker measurement

${ }^{C}$ Model adjusting for age at inflammatory biomarker measurement, fat percentage at biomarker measurement, ethnicity, birth weight, height age- and sex-specific $Z$-score, and maternal education

DLikelihood ratio test (LRT) $p$ value for whether the addition of inflammatory biomarker quartiles improved model fit relative to the model without indicators for inflammatory biomarker quartiles

EWald test $p$ value for log-transformed median within each quartile included as a continuous covariate in models adjusting for age at inflammatory biomarker measurement, fat percentage at biomarker measurement, ethnicity, birth weight, height age- and sex-specific $Z$-score, and maternal education

FInflammatory marker quartiles at Tanner 2: CRP (mg/L) [0.1-0.3], [0.4-0.7], [0.8-2.2], [2.3-15.7]; IL-6 (pg/mL) [0.3-0.8], [0.9-1.3], [1.4-2.2], [2.3-30.4]; TNFR2 (pg/mL) [944.4-1894.1], [1897.2-2169.0], [2171.9-2533.7], [2545.9-4902.5]. Inflammatory marker quartiles at Tanner 4: CRP (mg/L) [0.1-0.2], [0.2-0.5], [0.5-1.9], [2.0-18.9]; IL$6(\mathrm{pg} / \mathrm{mL})[0.3-0.8],[0.9-1.3],[1.4-2.1],[2.2-24.0] ;$ TNFR2 (pg/mL) [1106.7-1960.4], [1971.3-2271.1], [2275.8-2638.1], [2639.9-4753.3]

${ }^{*} p<0.05$

** $p<0.01$

${ }^{* * *} p<0.001$

associated with dense breast area, and the association with dense area persisted in fully adjusted models [46].

In our study, we observed disparate associations between specific inflammatory markers and age at menarche in our fully adjusted models. The robustness of these associations to adjustment for total body fat percentage suggests that inflammatory markers may be related to ovarian production of reproductive hormones. An increase in CRP levels at Tanner 4 was associated with longer relative time to menarche. Interestingly, a small $(n=25)$ study of Polish women reported that an earlier age at menarche was associated with higher levels of CRP in adulthood [47]. The inversion of this relation before and after menarche suggests that CRP may be associated with multiple facets of pubertal development. A cross-sectional study of US adolescents did not observe a significant association between CRP and self-reported pubertal status [48]. However, they did report a positive relation between pubertal status and IL- 6 and an inverse relation with TNF $\alpha$ among both males and females [48]. 
Table 4 Association between inflammatory marker measurement at Tanner 2 and Tanner 4 with relative time to menarche

\begin{tabular}{|c|c|c|c|c|}
\hline Inflammatory marker & Breast Tanner stage & $N$ & Events $^{\mathrm{D}}$ & $\begin{array}{l}\text { Relative time to menarche per } \\
\text { doubling of inflammatory } \\
\text { marker; time ratio }(95 \% \mathrm{Cl})\end{array}$ \\
\hline \multicolumn{5}{|l|}{ Age-adjusted model $^{A}$} \\
\hline \multirow[t]{2}{*}{ CRP } & Tanner 2 & 348 & 302 & $1.00(0.99-1.00)$ \\
\hline & Tanner 4 & 298 & 291 & $1.01(1.00-1.01)^{* * *}$ \\
\hline \multirow[t]{2}{*}{ IL-6 } & Tanner 2 & 381 & 335 & $0.99(0.99-1.00)^{*}$ \\
\hline & Tanner 4 & 298 & 291 & $1.00(1.00-1.01)$ \\
\hline \multirow[t]{2}{*}{ TNFR2 } & Tanner 2 & 381 & 335 & $0.97(0.95-0.99)^{*}$ \\
\hline & Tanner 4 & 298 & 291 & $1.00(0.98-1.02)$ \\
\hline \multicolumn{5}{|c|}{ Age and body fatness-adjusted model ${ }^{B}$} \\
\hline \multirow[t]{2}{*}{ CRP } & Tanner 2 & 347 & 301 & $1.00(0.99-1.00)$ \\
\hline & Tanner 4 & 298 & 291 & $1.01(1.00-1.01)^{* * *}$ \\
\hline \multirow[t]{2}{*}{ IL-6 } & Tanner 2 & 380 & 334 & $0.99(0.99-1.00)^{*}$ \\
\hline & Tanner 4 & 298 & 291 & $1.00(0.99-1.01)$ \\
\hline \multirow[t]{2}{*}{ TNFR2 } & Tanner 2 & 380 & 334 & $0.97(0.95-1.00)^{*}$ \\
\hline & Tanner 4 & 298 & 291 & $1.00(0.98-1.01)$ \\
\hline \multicolumn{5}{|c|}{ Multivariable-adjusted model $^{C}$} \\
\hline \multirow[t]{2}{*}{ CRP } & Tanner 2 & 336 & 291 & $1.00(0.99-1.00)$ \\
\hline & Tanner 4 & 291 & 284 & $1.01(1.00-1.01)^{* * *}$ \\
\hline \multirow[t]{2}{*}{ IL-6 } & Tanner 2 & 369 & 324 & $0.99(0.98-1.00)^{* *}$ \\
\hline & Tanner 4 & 291 & 284 & $1.00(0.99-1.01)$ \\
\hline \multirow[t]{2}{*}{ TNFR2 } & Tanner 2 & 369 & 324 & $0.98(0.96-1.00)^{*}$ \\
\hline & Tanner 4 & 291 & 284 & $1.00(0.98-1.02)$ \\
\hline
\end{tabular}

${ }^{A}$ Accelerated failure time model for time to menarche from birth adjusting for age at inflammatory biomarker measurement

${ }^{\mathrm{B}}$ Model adjusting for age at inflammatory biomarker measurement and fat percentage at biomarker measurement

${ }^{C}$ Model adjusting for age at inflammatory biomarker measurement, fat percentage at biomarker measurement, ethnicity, birth weight, height age- and sex-specific

Z-score, and maternal education

${ }^{D}$ Number of girls reaching menarche during follow-up period

${ }^{*} p<0.05$

${ }^{* *} p<0.01$

${ }^{* * *} p<0.001$

In our longitudinal study, we did not observe an association between TNFR2 levels and age at menarche, but higher IL-6 levels at Tanner 2 were associated with an earlier age of menarche, which is consistent with the US study. Reproductive hormones, including estrogen and testosterone, increase substantially during puberty and are known to influence immune responses and inflammatory pathways [49]. We did not detect this same association in Tanner 4, which may be due to the loss of very early developers for the analysis of associations with Tanner 4 inflammatory markers (left-censored individuals). Our sensitivity analysis, assessing the degree to which selection bias may be contributing to differences in the estimated associations with biomarker levels at Tanner 2 and 4, indicated that that the association estimates and inference among individuals with biomarker measurements at both time points did not differ from our primary results. Overall, our results suggest that the associations between reproductive hormones and specific inflammatory markers are not consistent between Tanner 2 and Tanner 4 stages. Prior epidemiologic studies among postmenopausal women have reported differential effect of estrogen on CRP and IL-6, suggesting disparate mechanisms of action [50].

These inflammatory markers have previously been associated with breast cancer risk, particularly among postmenopausal women. The Health Aging and Body Composition study of women aged 70-79 years old evaluated baseline inflammatory markers (e.g., IL6, TNFR $\alpha$, $\mathrm{CRP}$ ) and incident breast cancer events. Due to the limited number of cases $(n=30-33)$, the authors were unable to reject the null hypothesis for IL6 and TNFR $\alpha$ (0.95 (95\% CI 0.54-1.65); HR 0.70 (95\% CI 0.29-1.72)) but observed a possible positive association with CRP (HR 1.32 (95\% CI 0.91-1.93)) [6]. A meta-analysis similarly indicated that for each doubling of CRP concentration, risk of any breast cancer and postmenopausal breast cancer was elevated 7\% (95\% CI 2-12\%) and 6\% 
Table 5 Association of quartiles of inflammatory markers measured at Tanner 2 and Tanner 4 with relative time to menarche

\begin{tabular}{|c|c|c|c|c|c|c|c|c|}
\hline \multirow{2}{*}{$\begin{array}{l}\text { Inflammatory } \\
\text { marker }\end{array}$} & \multirow{2}{*}{$\begin{array}{l}\text { Breast } \\
\text { Tanner } \\
\text { stage }\end{array}$} & \multirow[b]{2}{*}{$N$} & \multirow[b]{2}{*}{ Events $^{G}$} & \multirow[b]{2}{*}{ LRT $p$ value ${ }^{\mathrm{D}}$} & \multicolumn{3}{|c|}{ Relative time to menarche compared to Q1; time ratio $(95 \% \mathrm{Cl})^{\mathrm{F}}$} & \multirow[b]{2}{*}{$p$ for Trend ${ }^{E}$} \\
\hline & & & & & Q2 & Q3 & Q4 & \\
\hline \multicolumn{9}{|c|}{ Age-adjusted model ${ }^{\mathrm{A}}$} \\
\hline \multirow[t]{2}{*}{ CRP } & Tanner 2 & 348 & 302 & 0.167 & $0.99(0.97-1.01)$ & $1.00(0.99-1.02)$ & $0.98(0.97-1.00)$ & 0.299 \\
\hline & Tanner 4 & 298 & 291 & $<0.0001$ & $0.98(0.97-1.00)^{*}$ & $1.01(1.00-1.02)$ & $1.02(1.01-1.04)^{* *}$ & $<0.001$ \\
\hline \multirow[t]{2}{*}{ IL-6 } & Tanner 2 & 381 & 335 & 0.532 & $1.00(0.98-1.02)$ & $1.00(0.98-1.02)$ & $0.99(0.97-1.01)$ & 0.157 \\
\hline & Tanner 4 & 298 & 291 & 0.463 & $1.01(0.99-1.02)$ & $1.01(1.00-1.03)$ & $1.01(1.00-1.03)$ & 0.157 \\
\hline \multirow[t]{2}{*}{ TNFR2 } & Tanner 2 & 381 & 335 & 0.103 & $1.01(0.99-1.03)$ & $1.00(0.98-1.02)$ & $0.98(0.96-1.00)$ & 0.047 \\
\hline & Tanner 4 & 298 & 291 & 0.217 & $1.01(1.00-1.03)$ & $1.01(0.99-1.02)$ & $1.00(0.98-1.02)$ & 0.666 \\
\hline \multicolumn{9}{|c|}{ Age and body fatness-adjusted model ${ }^{B}$} \\
\hline \multirow[t]{2}{*}{ CRP } & Tanner 2 & 347 & 301 & 0.280 & $0.99(0.97-1.01)$ & $1.00(0.99-1.02)$ & $0.99(0.97-1.01)$ & 0.451 \\
\hline & Tanner 4 & 298 & 291 & $<0.001$ & $0.98(0.97-1.00)^{*}$ & $1.01(0.99-1.02)$ & $1.02(1.00-1.03)^{*}$ & 0.001 \\
\hline \multirow[t]{2}{*}{ IL-6 } & Tanner 2 & 380 & 334 & 0.602 & $1.00(0.98-1.02)$ & $1.00(0.98-1.02)$ & $0.99(0.97-1.01)$ & 0.197 \\
\hline & Tanner 4 & 298 & 291 & 0.758 & $1.01(0.99-1.02)$ & $1.01(0.99-1.02)$ & $1.01(0.99-1.02)$ & 0.484 \\
\hline \multirow[t]{2}{*}{ TNFR2 } & Tanner 2 & 380 & 334 & 0.121 & $1.01(0.99-1.03)$ & $1.00(0.98-1.02)$ & $0.98(0.96-1.00)$ & 0.058 \\
\hline & Tanner 4 & 298 & 291 & 0.136 & $1.01(1.00-1.03)$ & $1.00(0.99-1.02)$ & $1.00(0.98-1.01)$ & 0.365 \\
\hline \multicolumn{9}{|c|}{ Multivariable-adjusted model $^{C}$} \\
\hline \multirow[t]{2}{*}{ CRP } & Tanner 2 & 336 & 291 & 0.260 & $0.99(0.97-1.01)$ & $1.01(0.99-1.02)$ & $0.99(0.97-1.01)$ & 0.839 \\
\hline & Tanner 4 & 291 & 284 & $<0.0001$ & $0.98(0.97-1.00)^{*}$ & $1.01(1.00-1.02)$ & $1.02(1.00-1.03)^{*}$ & $<0.001$ \\
\hline \multirow[t]{2}{*}{ IL-6 } & Tanner 2 & 369 & 324 & 0.048 & $1.00(0.98-1.02)$ & $1.00(0.98-1.02)$ & $0.97(0.95-0.99)^{* *}$ & 0.010 \\
\hline & Tanner 4 & 291 & 284 & 0.897 & $1.01(0.99-1.02)$ & $1.00(0.99-1.02)$ & $1.00(0.99-1.02)$ & 0.782 \\
\hline \multirow[t]{2}{*}{ TNFR2 } & Tanner 2 & 369 & 324 & 0.062 & $1.01(0.99-1.03)$ & $0.99(0.97-1.00)$ & $0.98(0.97-1.00)$ & 0.044 \\
\hline & Tanner 4 & 291 & 284 & 0.128 & $1.02(1.00-1.03)^{*}$ & $1.01(0.99-1.02)$ & $1.00(0.99-1.02)$ & 0.911 \\
\hline
\end{tabular}

${ }^{\mathrm{A}}$ Accelerated failure time model for time to menarche from birth adjusting for age at inflammatory biomarker measuremen

${ }^{\mathrm{B}}$ Model adjusting for age at inflammatory biomarker measurement and fat percentage at biomarker measurement

${ }^{C}$ Model adjusting for age at inflammatory biomarker measurement, fat percentage at biomarker measurement, ethnicity, birth weight, height age- and sex-specific $Z$-score, and maternal education

DLikelihood ratio test (LRT) $p$ value for whether the addition of inflammatory biomarker quartiles improved model fit relative to the model without indicators for inflammatory biomarker quartiles

EWald test $p$ value for log-transformed median within each quartile included as a continuous covariate in models adjusting for age at inflammatory biomarker measurement, fat percentage at biomarker measurement, ethnicity, birth weight, height age- and sex-specific $Z$-score, and maternal education

FInflammatory marker quartiles at Tanner 2: CRP (mg/L) [0.1-0.3], [0.4-0.7], [0.8-2.2], [2.3-15.7]; IL-6 (pg/mL) [0.3-0.8], [0.9-1.3], [1.4-2.2], [2.3-30.4]; TNFR2 (pg/mL) [944.4-1894.1], [1897.2-2169.0], [2171.9-2533.7], [2545.9-4902.5]. Inflammatory marker quartiles at Tanner 4: CRP (mg/L) [0.1-0.2], [0.2-0.5], [0.5-1.9], [2.0-18.9]; IL6 (pg/mL) [0.3-0.8], [0.9-1.3], [1.4-2.1], [2.2-24.0]; THFR2 (pg/mL) [1106.7-1960.4], [1971.3-2271.1], [2275.8-2638.1], [2639.9-4753.3]

${ }^{\mathrm{G}}$ Number of girls reaching menarche during follow-up period

${ }^{*} p<0.05$

${ }^{* *} p<0.01$

${ }^{* * *} p<0.001$

(95\% CI 1-11\%), respectively [51]. Another metaanalysis reviewing the association between obesityrelated adipocytes and breast cancer among Asian women found that increased levels of TNF $\alpha$ was associated with increased risk of postmenopausal breast cancer. Specifically, the concentrations of IL6 and TNF $\alpha$ were higher in women with breast cancer with pooled mean difference of 2.15 (95\% CI 1.64-2.66) and 1.70 (95\% CI 1.10-2.30), respectively. The review also emphasized that higher levels of TNF $\alpha$ were more prevalent among women with BMI $>25 \mathrm{~kg} / \mathrm{m}^{2}$ [52].

Strengths of our study include the prospective nature of the puberty cohort with repeated assessments of inflammatory markers, the unique availability of breast density assessments as a putative marker of future breast cancer risk, and the availability of well-measured correlated factors, such as body fatness. A limitation of our study is the number of participants that provided blood samples at Tanner 2 that did not consent to provide breast composition measurements at Tanner 4 . This loss to follow-up may partially account for the disparate associations between our inflammatory markers measured at Tanner 2 and Tanner 4 and our outcomes of interest. In spite of this limitation, our study adds valuable new insight into the relation between inflammatory markers during puberty and breast development. The observed increase in systemic inflammation with increased body size may contribute to breast tissue damage during a critical period of development. 


\section{Conclusions}

In conclusion, indicators of systemic inflammation during puberty were not associated with adolescent breast volume or breast density after adjustment for body fatness; however, such circulating inflammation biomarkers may affect the timing of onset of menarche. While the exact mechanism by which inflammation plays a role in breast density development warrants further study, the shared positive relationship between total body fat, breast size, and pro-inflammatory markers may have important implications for future cancer risk.

\section{Supplementary information}

Supplementary information accompanies this paper at https://doi.org/10. 1186/s13058-020-01338-y.

Additional file 1. Study population characteristics of girls with measured inflammatory markers at Tanner stage 2 but no breast composition measurements at Tanner stage 4.

Additional file 2. Study population characteristics of girls with measured inflammatory markers at Tanner stage 4 but no breast composition measurements at Tanner stage 4.

Additional file 3. Association of inflammatory markers measured at Tanner 2 and Tanner 4 with breast composition measured at Tanner 4; restricting to individuals for which inflammatory markers were measured at Tanner 2 and Tanner 4.

Additional file 4. Association of quartiles of inflammatory markers measured at Tanner 2 and Tanner 4 with breast composition measured at Tanner 4; restricting to individuals for which inflammatory markers were measured at Tanner 2 and Tanner 4.

Additional file 5. Association of inflammatory marker measurement at Tanner 2 and Tanner 4 with relative time to menarche; restricting to individuals for which inflammatory markers were measured at Tanner 2 and Tanner 4.

Additional file 6. Association of quartiles of inflammatory markers measured at Tanner 2 and Tanner 4 with relative time to menarche; restricting to individuals for which inflammatory markers were measured at Tanner 2 and Tanner 4.

\section{Abbreviations}

IL-6: Interleukin-6; TNFa: Tumor necrosis factor a; CRP: C-reactive protein; TNF: Tumor necrosis factor; TNFR2: Tumor necrosis factor receptor 2; SWAN: Study of Women's Health Across the Nation; GOCS: Growth and Obesity Cohort Study; INTA: Institute of Nutrition and Food Technology; Tanner: Tanner's rating scale; FGV: Breast fibroglandular volume; BV: Total breast volume; FGV\%: Fibroglandular volume; DXA: Dual-energy X-ray absorptiometry; hsCRP: High sensitivity C-reactive protein; WHO: World Health Organization; Cl: Confidence interval

\section{Acknowledgements}

The authors thank the Research Center on Food Environment and Prevention of Chronic Diseases (CIAPEC) team and the participants of the Growth and Obesity Cohort Study for providing the relevant data and biospecimen.

\section{Ethics of approval and consent to participate}

The study protocol was approved by the Ethics Committee of the Institute of Nutrition and Food Technology, University of Chile, and by the Institutional Review Board of UCLA. All parents and/or legal guardians gave signed informed consent prior to the data collection and children assent.

\section{Authors' contributions}

$\mathrm{KBM}$ and $\mathrm{AMB}$ designed the research plan. $\mathrm{KBM}, \mathrm{KK}$, and $\mathrm{AMB}$ conducted the statistical analyses. $\mathrm{KBM}, \mathrm{CEK}$, and $\mathrm{AMB}$ interpreted the results and co-wrote the manuscript. AP and CC acquired the clinical data analyzed in this study. JLS organized and processed the biospecimens. JS oversaw the breast composition measurements and interpretation. All authors provided critical revision of the manuscript for important intellectual content. KBM, CEK, and AMB have primary responsibility for final content. All authors read and approved the final manuscript.

\section{Funding}

This work was supported by Public Health Service grant R01CA158313 from the National Cancer Institute, National Institutes of Health, US Department of Health and Human Services (to KBM). Research reported in this publication was supported by the National Cancer Institute of the National Institutes of Health under Award Number K07CA225856 (to AMB) as well as FONDECYT 11170670, PCI Proyecto REDI170060, the World Cancer Research Fund (grant 2010/245). The content is solely the responsibility of the authors and does not necessarily represent the official views of the National Institutes of Health.

\section{Availability of data and materials}

The datasets used and/or analyzed during the current study are available from the corresponding author on reasonable request.

\section{Consent for publication}

Not applicable.

\section{Competing interests}

The authors declare that they have no competing interests.

\section{Author details}

${ }^{1}$ Department of Epidemiology, Fielding School of Public Health, University of California, 650 Charles Young Drive South, Room 71-264 CHS, Los Angeles, CA 90095, USA. ${ }^{2}$ Institute for Prevention and Cancer Epidemiology, Faculty of Medicine and Medical Center, University of Freiburg, Freiburg im Breisgau, Germany. ${ }^{3}$ Department of Biostatistics, Fielding School of Public Health, University of California, Los Angeles, CA, USA. ${ }^{4}$ Institute of Nutrition and Food Technology, University of Chile, Santiago, Chile. ${ }^{5}$ Department of Nutrition, Diabetes and Metabolism, School of Medicine, Pontificia Universidad Católica de Chile, Santiago, Chile. ${ }^{6}$ Population Sciences in the Pacific Program (Cancer Epidemiology), University of Hawai'i Cancer Center, University of Hawai'i, Honolulu, HI, USA.

Received: 3 February 2020 Accepted: 7 September 2020

Published online: 01 October 2020

\section{References}

1. Cabodi S, Taverna D. Interfering with inflammation: a new strategy to block breast cancer self-renewal and progression? Breast Cancer Res. 2010;12:305.

2. Coussens LM, Werb Z. Inflammation and cancer. Nature. 2002:420:860-7.

3. Jiang $X$, Shapiro DJ. The immune system and inflammation in breast cancer. Mol Cell Endocrinol. 2014;382:673-82.

4. Purohit A, Newman SP, Reed MJ. The role of cytokines in regulating estrogen synthesis: implications for the etiology of breast cancer. Breast Cancer Res. 2002:4:65

5. Boyd NF, Martin LJ, Bronskill M, Yaffe MJ, Duric N, Minkin S. Breast tissue composition and susceptibility to breast Cancer. J Natl Cancer Inst. 2010; 102:1224-37.

6. Il'yasova D, Colbert LH, Harris TB, Newman AB, Bauer DC, Satterfield S, et al. Circulating levels of inflammatory markers and cancer risk in the health aging and body composition cohort. Cancer Epidemiol Prev Biomark. 2005; 14:2413-8.

7. Hanna M, Dumas I, Orain M, Jacob S, Têtu B, Sanschagrin F, et al. Association between expression of inflammatory markers in normal breast tissue and mammographic density among premenopausal and postmenopausal women. Menopause. 2017:24:524-35.

8. Gyamfi J, Eom M, Koo J-S, Choi J. Multifaceted roles of interleukin- 6 in adipocyte-breast cancer cell interaction. Transl Oncol. 2018;11:275-85.

9. Yang S, Wang J, Brand DD, Zheng SG. Role of TNF-TNF receptor 2 signal in regulatory T cells and its therapeutic implications. Front Immunol. 2018;9: 784. 
10. Sheng Y, Li F, Qin Z. TNF receptor 2 makes tumor necrosis factor a friend of tumors. Front Immunol. 2018;9 Available from: https://www.ncbi.nlm.nih. gov/pmc/articles/PMC5985372/. [cited 2019 Jun 18].

11. Shrotriya S, Walsh D, Bennani-Baiti N, Thomas S, Lorton C. C-reactive protein is an important biomarker for prognosis tumor recurrence and treatment response in adult solid tumors: a systematic review. Zhang L, editor. PLoS One. 2015;10:e0143080

12. Zhao H, Zhou L, Shangguan AJ, Bulun SE. Aromatase expression and regulation in breast and endometrial cancer. J Mol Endocrinol. 2016;57:R1933.

13. Zhao $Y$, Nichols JE, Valdez R, Mendelson CR, Simpson ER. Tumor necrosis factor-alpha stimulates aromatase gene expression in human adipose stromal cells through use of an activating protein-1 binding site upstream of promoter 1.4. Mol Endocrinol. 1996;10:1350-7.

14. Allin $\mathrm{KH}$, Nordestgaard BG, Flyger $\mathrm{H}$, Bojesen SE. Elevated pre-treatment levels of plasma C-reactive protein are associated with poor prognosis after breast cancer: a cohort study. Breast Cancer Res. 2011;13:R55.

15. Makboon K, Gold EB, Harvey DJ, Butler LM, Habel LA. Association between high-sensitivity C-reactive protein (hsCRP) and change in mammographic density over time in the SWAN mammographic density subcohort. Cancer Causes Control. 2015;26:431-42

16. Bodicoat DH, Schoemaker MJ, Jones ME, McFadden E, Griffin J, Ashworth A, et al. Timing of pubertal stages and breast cancer risk: the breakthrough generations study. Breast Cancer Res. 2014;16:R18.

17. Biro FM, Greenspan LC, Galvez MP. Puberty in girls of the 21 st century. J Pediatr Adolesc Gynecol. 2012;25:289-94.

18. Shepherd JA, Malkov S, Fan B, Laidevant A, Novotny R, Maskarinec G. Breast density assessment in adolescent girls using dual-energy $\mathrm{X}$-ray absorptiometry: a feasibility study. Cancer Epidemiol Prev Biomark. 2008;17: 1709-13.

19. Houghton LC, Jung S, Troisi R, LeBlanc ES, Snetselaar LG, Hylton NM, et al. Pubertal timing and breast density in young women: a prospective cohort study. Breast Cancer Res. 2019;21 Available from: https://breast-cancerresearch.biomedcentral.com/articles/10.1186/s13058-019-1209-x. [cited 2020 Jan 7].

20. Binder AM, Corvalan C, Pereira A, Calafat AM, Ye X, Shepherd J, et al. Prepubertal and pubertal endocrine-disrupting chemical exposure and breast density among Chilean adolescents. Cancer Epidemiol Prev Biomark. 2018;27:1491-9

21. Kain J, Corvalán C, Lera L, Galván M, Uauy R. Accelerated growth in early life and obesity in preschool Chilean children. Obesity. 2009;17:1603-8.

22. Pereira A, Garmendia ML, González D, Kain J, Mericq V, Uauy R, et al. Breast bud detection: a validation study in the Chilean growth obesity cohort study. BMC Womens Health. 2014;14 Available from: http:// bmcwomenshealth.biomedcentral.com/articles/10.1186/1472-6874-14-96. [cited 2019 Sep 27].

23. Shepherd JA, Herve L, Landau J, Fan B, Kerlikowske K, Cummings SR. Clinical comparison of a novel breast DXA technique to mammographic density. Med Phys. 2006;33:1490-8.

24. Adiotomre E, Summers L, Allison A, Walters SJ, Digby M, Broadley P, et al. Diagnostic accuracy of DXA compared to conventional spine radiographs for the detection of vertebral fractures in children. Eur Radiol. 2017;27:2188-99.

25. Costa M, Garmendia ML, Corvalán C, Reyes M. The presence and duration of overweight are associated with low-grade inflammation in prepubertal Chilean children. Metab Syndr Relat Disord. 2016;14:449-54.

26. Corvalán C, Uauy R, Mericq V. Obesity is positively associated with dehydroepiandrosterone sulfate concentrations at $7 \mathrm{y}$ in Chilean children of normal birth weight. Am J Clin Nutr. 2013;97:318-25.

27. de Onis M, Onyango AW, Borghi E, Siyam A, Nishida C, Siekmann J. Development of a WHO growth reference for school-aged children and adolescents. Bull World Health Organ. 2007:85:660-7.

28. Cediel G, Corvalán C, Aguirre C, de Romaña DL, Uauy R. Serum 25Hydroxyvitamin D associated with indicators of body fat and insulin resistance in prepubertal Chilean children. Int J Obes. 2016;40:147-52.

29. Fernández M, Pereira A, Corvalán C, Mericq V. Precocious pubertal events in Chilean children: ethnic disparities. J Endocrinol Investig. 2019:42:385-95.

30. Pettersson-Pablo P, Nilsson TK, Breimer LH, Hurtig-Wennlöf A. Body fat percentage is more strongly associated with biomarkers of low-grade inflammation than traditional cardiometabolic risk factors in healthy young adults - the lifestyle, biomarkers, and atherosclerosis study. Scand J Clin Lab Invest. 2019;79:182-7.
31. Schmeer KK, Tarrence J. Racial/ethnic disparities in inflammation: evidence of weathering in childhood? J Health Soc Behav. 2018;59:411-28.

32. Boscaini C, Pellanda LC. Birth weight, current anthropometric markers, and high sensitivity C-reactive protein in Brazilian school children. J Obes. 2015, 2015; Available from: https://www.ncbi.n/m.nih.gov/pmc/articles/PMC43833 89/. [cited 2020 Jan 7].

33. Kaplowitz PB. Link between body fat and the timing of puberty. Pediatrics. 2008;121:S208-17.

34. Kubo A, Deardorff J, Laurent CA, Ferrara A, Greenspan LC, Quesenberry CP, et al. Associations between maternal obesity and pregnancy hyperglycemia and timing of puberty onset in adolescent girls: a population-based study. Am J Epidemiol. 2018;187:1362-9.

35. Hiatt RA, Stewart SL, Hoeft KS, Kushi LH, Windham GC, Biro FM, et al. Childhood socioeconomic position and pubertal onset in a cohort of multiethnic girls: implications for breast cancer. Cancer Epidemiol Biomark Prev. 2017;26:1714-21.

36. Kusano AS, Trichopoulos D, Terry KL, Chen WY, Willett WC, Michels KB. A prospective study of breast size and premenopausal breast cancer incidence. Int J Cancer. 2006;118:2031-4.

37. Ellulu MS, Patimah I, Khaza'ai H, Rahmat A, Abed Y. Obesity and inflammation: the linking mechanism and the complications. Arch Med Sci AMS. 2017:13:851-63.

38. Deng T, Lyon CJ, Bergin S, Caligiuri MA, Hsueh WA. Obesity, inflammation, and cancer. Annu Rev Pathol. 2016;11:421-49.

39. Garcia-Hermoso A, Agostinis-Sobrinho C, Mota J, Santos RM, Correa-Bautista JE, Ramírez-Vélez R. Adiposity as a full mediator of the influence of cardiorespiratory fitness and inflammation in schoolchildren: the FUPRECOL study. Nutr Metab Cardiovasc Dis. 2017;27:525-33.

40. Kershaw EE, Flier JS. Adipose tissue as an endocrine organ. J Clin Endocrinol Metab. 2004;89:2548-56.

41. Tam CS, Clément K, Baur LA, Tordjman J. Obesity and low-grade inflammation: a paediatric perspective. Obes Rev. 2010;11:118-26.

42. Chiarelli F, Marcovecchio ML. Insulin resistance and obesity in childhood. Eur J Endocrinol. 2008;159(Suppl 1):S67-74.

43. Dowd JB, Zajacova A, Aiello AE. Predictors of inflammation in U.S. children aged 3-16 years. Am J Prev Med. 2010;39:314-20.

44. Rehman K, MSH A. Mechanisms of inflammatory responses and development of insulin resistance: how are they interlinked? J Biomed Sci. 2016;23 Available from: https://www.ncbi.nlm.nih.gov/pmc/articles/PMC5135 788/. [cited 2019 Sep 27].

45. Wärnberg J, Marcos A. Low-grade inflammation and the metabolic syndrome in children and adolescents. Curr Opin Lipidol. 2008;19:11-5.

46. Fang CY, Egleston BL, Byrne C, Bohr GS, Pathak HB, Godwin AK, et al. Inflammation and breast density among female Chinese immigrants: exploring variations across neighborhoods. Cancer Causes Control. 2019;30: 1113-26.

47. Clancy KBH, Klein LD, Ziomkiewicz A, Nenko I, Jasienska G, Bribiescas RG. Relationships between biomarkers of inflammation, ovarian steroids, and age at menarche in a rural Polish sample. Am J Hum Biol Off J Hum Biol Counc. 2013:25:389-98.

48. Stumper A, Moriarity DP, Coe CL, Ellman LM, Abramson LY, Alloy LB. Pubertal Status and Age are Differentially Associated with Inflammatory Biomarkers in Female and Male Adolescents. J Youth Adolesc. 2020;49: 1379-92.

49. Shirtcliff EA, Dahl RE, Pollak SD. Pubertal development: correspondence between hormonal and physical development. Child Dev. 2009;80:327-37.

50. Lakoski SG, Herrington DM. Effects of hormone therapy on C-reactive protein and IL-6 in postmenopausal women: a review article. Climacteric J Int Menopause Soc. 2005:8:317-26.

51. Chan DSM, Bandera EV, Greenwood DC, Norat T. Circulating C-reactive protein and breast cancer risk--systematic literature review and metaanalysis of prospective cohort studies. Cancer Epidemiol Biomark Prev. 2015; 24:1439-49.

52. Gui Y, Pan Q, Chen X, Xu S, Luo X, Chen L. The association between obesity related adipokines and risk of breast cancer: a meta-analysis. Oncotarget. 2017:8:75389-99.

\section{Publisher's Note}

Springer Nature remains neutral with regard to jurisdictional claims in published maps and institutional affiliations. 